\title{
A phase 2a randomized clinical trial of intravenous vedolizumab for the treatment of steroid-refractory intestinal acute graft-versus-host disease
}

\author{
Yngvar Fløisand $\mathbb{D}^{1,2} \cdot$ Mark A. Schroeder ${ }^{3} \cdot$ Patrice Chevallier $^{4} \cdot$ Dominik Selleslag $^{5} \cdot$ Steven Devine $^{6}$.

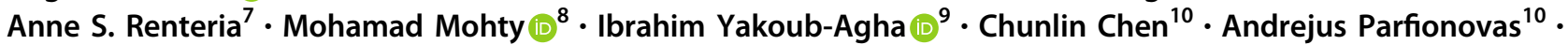 \\ Syed Quadri ${ }^{10} \cdot$ Johan Jansson $^{10} \cdot$ Mona Akbari $^{10} \cdot$ Yi-Bin Chen $\mathbb{1}^{11}$
}

Received: 21 August 2020 / Revised: 23 April 2021 / Accepted: 12 May 2021 / Published online: 9 June 2021

(c) The Author(s) 2021. This article is published with open access

\begin{abstract}
Steroid-refractory (SR) acute graft-versus-host disease (aGvHD) remains a significant complication after allogeneic hematopoietic cell transplantation. Systemic corticosteroids are first-line therapy for aGvHD, but apart from ruxolitinib, there are no approved treatments for SR aGvHD. Vedolizumab is approved for treatment of ulcerative colitis and Crohn's disease, and may be effective for treatment of SR intestinal aGvHD. We conducted a phase 2a trial (NCT02993783) to evaluate the clinical efficacy, tolerability, and safety of vedolizumab 300 and $600 \mathrm{mg}$ for SR intestinal aGvHD. This study was terminated before full enrollment was completed because early results failed to demonstrate positive proof-of-concept in efficacy. Before termination, 17 participants had enrolled and an early response in intestinal aGvHD was observed in 11 and eight participants at days 15 and 28, respectively. All adverse events observed were consistent with those expected in a population with SR intestinal aGvHD. Overall, vedolizumab did not meet the primary efficacy endpoint (overall response at day 28), likely owing to premature study drug discontinuation, lack of efficacy, and the competing risks inherent with a population with advanced SR intestinal aGvHD. Nevertheless, this study provides valuable insights into the considerations needed when conducting studies in patients with SR intestinal aGvHD.
\end{abstract}

\section{Introduction}

Allogeneic hematopoietic cell transplantation is an important and potentially curative therapy for many hematologic

Supplementary information The online version contains supplementary material available at https://doi.org/10.1038/s41409021-01356-0.

Yngvar Fløisand

yfloisan@ous-hf.no

1 Department of Haematology, Oslo University Hospital, Rikshospitalet, Oslo, Norway

2 Centre for Cancer Cell Reprogramming, Department of Molecular Cell Biology, Oslo University Hospital, Oslo, Norway

3 Division of Oncology, Washington University School of Medicine, St. Louis, MO, USA

4 CHU de l'Hôtel-Dieu, service d'hématologie clinique, place A. Ricordeau, Nantes, France

5 AZ Sint-Jan Brugge-Oostende, Brugge, Belgium malignancies, but carries the significant risk of graft-versushost disease (GvHD) [1, 2]. Acute GvHD (aGvHD), which generally occurs in the first few months after allogeneic hematopoietic stem cell transplantation, typically involves the skin, liver, and/or intestine [2-4].

After conventional allogeneic hematopoietic cell transplantation, the incidence of aGvHD ranges from $10 \%$ to $80 \%$ (dependent on certain clinical risk factors) in patients receiving T-cell-replete allogeneic grafts, despite standard measures of prophylaxis [5-7].

6 Center for International Blood and Marrow Transplant Research (CIMBTR) Minneapolis Campus, Minneapolis, MN, USA

7 Hematology Oncology, Cellular and Gene Therapy, ICON Clinical Research Service, New York, NY, USA

8 Hematology Department, AP-HP, Hôpital Saint-Antoine, Sorbonne Université, and INSERM UMRs 938, Paris, France

9 CHU de Lille, Univ Lille, INSERM U1286, Infinite, Lille, France

10 Millennium Pharmaceuticals Inc, Cambridge, MA, USA

11 Hematopoietic Cell Transplant and Cellular Therapy Program, Massachusetts General Hospital, Boston, MA, USA 
First-line therapy for patients who develop aGvHD is systemic corticosteroids, but these are effective only in $\sim 50 \%$ of patients, with durable responses observed in only one-third $[8,9]$. Apart from ruxolitinib, which is associated with cytopenias, there are no other approved treatments for steroidrefractory (SR) aGvHD, which is associated with historical 2-year survival rates of $<20 \%$ [10-12]. Furthermore, there are no targeted treatments for intestinal aGvHD, for the majority of morbidity and mortality associated with SR aGvHD [13].

Vedolizumab, a humanized immunoglobulin G1 monoclonal antibody directed against the $\alpha 4 \beta 7$ integrin expressed on lymphocytes and implicated in gastrointestinal (GI) trafficking, was developed for the treatment of moderate-severe ulcerative colitis and Crohn's disease [14-16]. Vedolizumab inhibits the interaction between $\alpha 4 \beta 7$ integrin and the mucosal addressin cell adhesion molecule-1 (MAdCAM-1), preventing the migration of gut-homing leukocytes into the GI mucosa [17-20]. Preclinical studies have suggested that blocking this interaction may prevent intestinal aGvHD, and multiple series have suggested that vedolizumab may have efficacy for the treatment of SR intestinal aGvHD [21-23].

Here we present data from a phase 2 a clinical trial (NCT02993783), which prospectively investigated the clinical efficacy, tolerability, and safety of vedolizumab for the treatment of SR intestinal aGvHD.

\section{Methods}

\section{Study design}

This was a phase $2 \mathrm{a}$, randomized, parallel, open-label study with two dose groups evaluating the clinical efficacy, tolerability, and safety of vedolizumab (Hospira McPherson, KS, USA) IV for treatment of SR intestinal aGvHD.

\section{Recruitment and consent}

Participants were recruited and enrolled from the investigator's practice or on referrals from other physicians. If alternative recruitment strategies were employed (such as advertisement) these were reviewed by the institutional review board (IRB)/independent ethics committee (IEC). All participants provided written informed consent before any study-required procedures were conducted, unless such procedure formed part of their standard of care.

\section{Eligibility criteria}

Participants were 18 years or older, had undergone a first allogeneic hematopoietic cell transplant, and had developed SR intestinal aGvHD (worsening or no improvement after
5-7 days of methylprednisolone $2 \mathrm{mg} / \mathrm{kg} /$ day or equivalent, or lack of a complete response after 14 days of methylprednisolone $2 \mathrm{mg} / \mathrm{kg} /$ day or equivalent (Supplementary Table 1)). Participants who developed intestinal aGvHD while receiving systemic therapy for other aGvHD organ manifestations were also eligible after 5-7 days, even if intestinal aGvHD had not been present for the entire duration. Participants were required to have Grade B-D aGvHD with intestinal involvement and an Eastern Cooperative Oncology Group (ECOG) Performance Status of 0-3. Patients who had received any systemic agents beyond corticosteroids for the treatment of aGvHD were excluded. Detailed inclusion and exclusion criteria are listed in Supplementary Table 2.

\section{Treatments}

The intravenous (IV) doses and schedule of vedolizumab were selected based on those approved for the treatment of ulcerative colitis and Crohn's disease (300 mg IV every 8 weeks) [14]. Because SR intestinal aGvHD may require higher doses of vedolizumab to achieve target saturation, owing to hypoalbuminemia, a dose of vedolizumab $600 \mathrm{mg}$ IV was also evaluated [14, 16, 24, 25].

Participants were randomized $1: 1$ to receive 300 or 600 mg doses of vedolizumab IV on days 1, 15, 43, 71, and 99 on the basis of evidence from a previous case series [26]. Investigators used an interactive voice or web response technology system at screening to obtain the participant study number and randomize them to receive 300 or $600 \mathrm{mg}$ doses of vedolizumab. Participants who responded to, and tolerated all five planned doses of vedolizumab, and who developed recurrent symptoms of intestinal aGvHD following discontinuation of therapy were permitted to receive additional doses of vedolizumab for up to 1 year from the first dose of the study drug (extension phase). Other medications considered necessary for the safety and wellbeing of the participant were permitted at the discretion of the investigator.

\section{Sample size}

It was intended that after ten participants had enrolled in each dose group and had been followed through day 28, safety, tolerability, efficacy, and pharmacokinetic (PK) results would be assessed to determine an appropriate dose for subsequent participants. The group at the chosen dose would have been expanded by approximately 18 additional participants to assess the tolerability and efficacy of vedolizumab further (approximately 38 patients in total were planned for enrollment). However, early results failed to demonstrate a positive proof-of-concept in efficacy (described below) and the trial was terminated early. 


\section{Study endpoints}

The primary endpoints of the study were overall response (OR) (defined as either a partial response, a very good partial response, or a complete response-Supplementary Table 1) at day 28 and incidence of treatment-emergent serious adverse events (TEAEs) following the administration of the first dose of vedolizumab to day 28 .

Secondary endpoints included overall survival at 6 and 12 months, complete response at day 28 , intestinal OR at day 28, and TEAEs and/or serious adverse events following the first dose of vedolizumab through 18 weeks after the last dose of vedolizumab (treatment exposure period). PK characterization of vedolizumab and the presence of antivedolizumab antibodies at baseline and at the end of the exposure period were also performed, and durable intestinal OR rate (intestinal OR achieved at day 28 and maintained OR at day 43) was evaluated as an exploratory endpoint.

All participants were planned to be followed for overall survival every 3 months until death, withdrawal of consent, termination of study by the sponsor, or for a maximum of 1 year after the last subject was enrolled in the study.

\section{Measurements}

Severity of aGvHD was assessed at each visit using the Blood and Marrow Transplant Clinical-Trials Network (BMT CTN)-modified International Blood and Marrow Transplant Research (IBMTR) database index (Supplementary Table 3) [27, 28]. The severity (Stage $0-4$ ) of skin, liver, and intestinal GvHD was based on aGvHD clinicalstage criteria.

Blood samples for PK analysis of vedolizumab were collected as per the schedule outlined in Supplementary Table 4. Vedolizumab serum concentrations were measured by enzyme-linked immunosorbent assay with a validated range of $0.20-8.00 \mu \mathrm{g} / \mathrm{mL}$. Immunogenicity was evaluated using blood sampling for anti-vedolizumab antibodies in pre-dose serum on day 1 , and on days 22,71 , and 99.

\section{Statistics}

A Bayesian statistical approach was planned for efficacy evaluation. An interim analysis was planned when all participants had reached day 28 (10 participants in each group). A positive proof-of-concept in efficacy was considered if the posterior probability of day 28 OR rate $>54 \%$ was at least $85 \%$ and day 28 OR rate being at least $70 \%$ was at least $75 \%$. Early review of patient-level data was performed ahead of the planned analysis to assess safety, PK, and efficacy data in order to facilitate decision-making in the study.

Enrolled participants who received any dose of study drug comprised the safety analysis set. The efficacy analysis set included all participants from the safety analysis set who had a baseline efficacy assessment and at least one postbaseline efficacy assessment. The PK analysis set comprised all participants in the safety analysis set who had at least one post-dose PK sample collected.

Approximately 38 patients were planned for enrollment. The sample size was based on clinical considerations and considered sufficient to determine a recommended dose and regimen and to describe the efficacy, tolerability, and safety of vedolizumab IV.

\section{Results}

\section{Study population}

On the basis of an early review of patient-level data and the totality of evidence available, it was concluded that the ability to evaluate overall efficacy was impaired owing to (a) the competing risks in the population, (b) premature discontinuation of study drug, and (c) observed mortality. It was determined that the proportion of participants achieving the primary efficacy outcome was not expected to reach $54 \%$ and the study was terminated early (9 May 2018) by the sponsor. Participants were recruited and followed from 28 April 2017 until study termination.

Before termination, 17 participants were enrolled and treated at 11 sites in Belgium, France, Norway, and the USA (Fig. 1). Of these, 13 participants completed day 28, four participants $(23.5 \%)$ completed all five planned doses of vedolizumab (two from each dose group), and one participant entered the extension phase and received two additional doses of vedolizumab $600 \mathrm{mg}$. The median number of doses in each treatment group was 2 .

\section{Demographics and disease characteristics}

Baseline characteristics are presented in Table 1. Overall median age was 57 years (range, 34-74 years), with four participants $(23.5 \%)$ aged 65 years or older. Five participants (29.4\%) had a baseline ECOG Performance Status of 3.

A total of 12 participants (70.6\%) had isolated intestinal $\mathrm{aGvHD}$, four (23.5\%) had skin and intestinal involvement, and one (5.9\%) had liver and intestinal involvement. Twelve participants $(70.6 \%)$ had Grade C or D aGvHD (eight Grade $\mathrm{C}$ aGvHD, four Grade D aGvHD). At baseline, 13 participants $(76.5 \%)$ had Stage 2 or greater intestinal aGvHD (one Stage 2, eight Stage 3, four Stage 4).

\section{Individual participant data}

Baseline disease characteristics and outcomes for each participant are presented in Supplementary Table 5A-C. A 


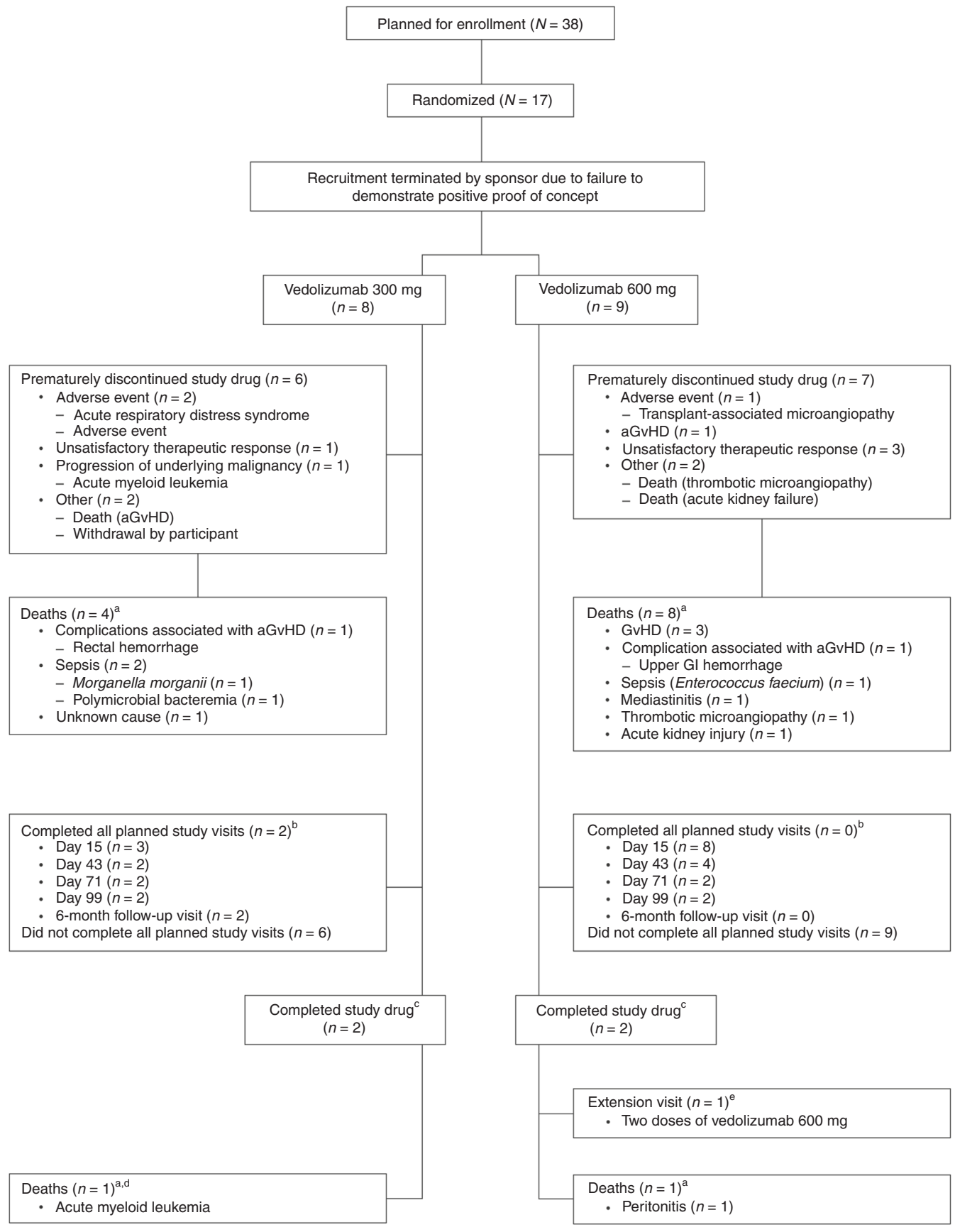

Fig. 1 Flow diagram of participants. Data presented are from the

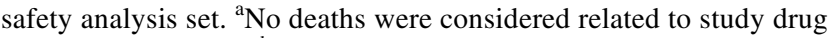
by the investigator. ${ }^{\mathrm{b}}$ Completed all planned study visits includes participants who completed all protocol required assessments and did not discontinue the study early. ${ }^{\mathrm{c}}$ Completed study drug includes participants who completed all five planned doses. ${ }^{\mathrm{d}}$ An additional participant in the vedolizumab IV $300 \mathrm{mg}$ group died after the end of the treatment-emergent period (i.e., 18 weeks after the last dose of study drug). ${ }^{\mathrm{e}}$ Upon review and agreement by the medical monitor, participants who responded to and tolerated all five planned doses of

vedolizumab and who developed recurrent symptoms of intestinal aGvHD following discontinuation of therapy (i.e., after the fifth dose) could enter an extension phase to receive vedolizumab $300 \mathrm{mg}$ every 2 weeks for two doses followed by every 4 weeks for up to 1 year from the first dose of study drug. A dose other than $300 \mathrm{mg}$ and/or a frequency of administration other than every 4 weeks may have been chosen based on accumulating safety, tolerability, efficacy, and pharmacokinetic results. $a G v H D$ acute graft-versus-host disease, $I V$ intravenous. 
Table 1 Baseline characteristics.

\begin{tabular}{|c|c|c|c|}
\hline & \multicolumn{3}{|c|}{ Number of participants $n,(\%)$} \\
\hline & $\begin{array}{l}\text { Vedolizumab } \\
300 \mathrm{mg} \\
(n=8)\end{array}$ & $\begin{array}{l}\text { Vedolizumab } \\
600 \mathrm{mg} \\
(n=9)\end{array}$ & $\begin{array}{l}\text { Total } \\
(N=17)\end{array}$ \\
\hline \multicolumn{4}{|l|}{ Age (years) ${ }^{\mathrm{a}}$} \\
\hline Mean (SD) & $54.1(10.45)$ & $59.0(10.87)$ & $56.7(10.64)$ \\
\hline $\begin{array}{l}\text { Minimum, } \\
\text { maximum }\end{array}$ & 40,74 & 34,70 & 34,74 \\
\hline \multicolumn{4}{|l|}{ Sex, $n(\%)$} \\
\hline Male & $2(25.0)$ & $5(55.6)$ & $7(41.2)$ \\
\hline Female & $6(75.0)$ & $4(44.4)$ & $10(58.8)$ \\
\hline \multicolumn{4}{|c|}{ ECOG performance status $n,(\%)^{\mathrm{b}}$} \\
\hline 0 & $2(25.0)$ & 0 & $2(11.8)$ \\
\hline 1 & $3(37.5)$ & $2(22.2)$ & $5(29.4)$ \\
\hline 2 & $1(12.5)$ & $4(44.4)$ & $5(29.4)$ \\
\hline 3 & $2(25.0)$ & $3(33.3)$ & $5(29.4)$ \\
\hline 4 & 0 & 0 & 0 \\
\hline \multicolumn{4}{|c|}{ aGvHD characteristics, grade ${ }^{c}$} \\
\hline Grade A & 0 & 0 & 0 \\
\hline Grade B & $2(25.0)$ & $3(33.3)$ & $5(29.4)$ \\
\hline Grade C & $4(50.0)$ & $4(44.4)$ & $8(47.1)$ \\
\hline Grade D & $2(25.0)$ & $2(22.2)$ & $4(23.5)$ \\
\hline \multicolumn{4}{|c|}{ aGvHD characteristics, involvements } \\
\hline Intestinal only & $7(87.5)$ & $5(55.6)$ & $12(70.6)$ \\
\hline Skin and intestinal & $1(12.5)$ & $3(33.3)$ & $4(23.5)$ \\
\hline Liver and intestinal & 0 & $1(11.1)$ & $1(5.9)$ \\
\hline $\begin{array}{l}\text { Skin, liver, and } \\
\text { intestinal }\end{array}$ & 0 & 0 & 0 \\
\hline \multicolumn{4}{|c|}{ aGvHD characteristics, GI tract ${ }^{\mathrm{d}}$} \\
\hline Stage 0 & 0 & 0 & 0 \\
\hline Stage 1 & $1(12.5)$ & $3(33.3)$ & $4(23.5)$ \\
\hline Stage 2 & $1(12.5)$ & 0 & $1(5.9)$ \\
\hline Stage 3 & $4(50.0)$ & $4(44.4)$ & $8(47.1)$ \\
\hline Stage 4 & $2(25.0)$ & $2(22.2)$ & $4(23.5)$ \\
\hline \multicolumn{4}{|l|}{ Conditioning regimen } \\
\hline $\begin{array}{l}\text { Myeloablative } \\
\text { transplant }\end{array}$ & $4(50.0)$ & $3(33.3)$ & $7(41.2)$ \\
\hline $\begin{array}{l}\text { Non-myeloablative } \\
\text { or reduced-intensity } \\
\text { transplant }\end{array}$ & $4(50.0)$ & $6(66.7)$ & $10(58.8)$ \\
\hline \multicolumn{4}{|l|}{ Stem cell source } \\
\hline Bone marrow & $1(12.5)$ & $1(11.1)$ & $2(11.8)$ \\
\hline Peripheral blood & $7(87.5)$ & $8(88.9)$ & $15(88.2)$ \\
\hline \multicolumn{4}{|l|}{ HLA compatibility } \\
\hline Matched & $6(75.0)$ & $6(66.7)$ & $12(70.6)$ \\
\hline Mismatched & $1(12.5)$ & $1(11.1)$ & $2(11.8)$ \\
\hline Haploidentical & $1(12.5)$ & $2(22.2)$ & $3(17.6)$ \\
\hline
\end{tabular}

Table 1 (continued)

\begin{tabular}{llll}
\hline & \multicolumn{3}{l}{ Number of participants $n,(\%)$} \\
\cline { 2 - 4 } & Vedolizumab & Vedolizumab & Total \\
$300 \mathrm{mg}$ & $600 \mathrm{mg}$ & $(N=17)$ \\
$(n=8)$ & $(n=9)$ & \\
\hline
\end{tabular}

CMV IgG antibody mismatch, donor/recipient

\begin{tabular}{llll} 
Positive/positive & $2(25.0)$ & $1(11.1)$ & $3(17.6)$ \\
Positive/negative & $2(25.0)$ & $2(22.2)$ & $4(23.5)$ \\
Negative/positive & $2(25.0)$ & $2(22.2)$ & $4(23.5)$ \\
Negative/negative & $2(25.0)$ & $4(44.4)$ & $6(35.3)$ \\
\hline
\end{tabular}

CMV cytomegalovirus, ECOG Eastern Cooperative Oncology Group, $I g G$ immunoglobulin G, $G I$ gastrointestinal, $G v H D$ graft-versus-host disease, $H L A$ human leukocyte antigen, $S D$ standard deviation.

${ }^{\mathrm{a}}$ Age at the date of informed consent.

$\mathrm{b}(0)$ Fully active, able to carry on all predisease performance without restriction, (1) restricted in physically strenuous activity, but ambulatory and able to carry out work of a light or sedentary nature (e.g., light housework, office work), (2) ambulatory and capable of all self-care, but unable to carry out any work activities, up and about more than $50 \%$ of waking hours, (3) capable of only limited self-care, confined to bed or chair more than $50 \%$ of waking hours, (4) completely disabled, cannot carry on any self-care, totally confined to bed or chair.

${ }^{\mathrm{c}}$ Grades were derived using the Blood and Marrow Transplant Clinical Trials Network (BMT CTN)-modified International Blood and Marrow Transplant Research (IBMTR) database index.

${ }^{\mathrm{d}}$ Stage is based on aGvHD clinical stage criteria (Supplementary Table 3).

summary of the main participant characteristics and outcomes are outlined in Fig. 2.

\section{Discontinuation of study drug}

Thirteen participants (76.5\%) discontinued the study drug prematurely (Fig. 1). Five of these 13 participants (38.5\%) discontinued due to progression of aGvHD $(n=$ 1) or because of an unsatisfactory therapeutic response $(n=4)$. Four participants $(30.8 \%)$ discontinued owing to 'other' reasons, including death $(n=3)$ and withdrawal $(n=1)$, three participants $(23.1 \%)$ owing to a TEAE (thrombotic microangiopathy, acute respiratory distress syndrome, and adverse event, none of which were vedolizumab-related), and one participant (7.7\%) owing to progression of an underlying malignancy (relapse of acute myeloid leukemia).

Of those who discontinued for reasons other than unsatisfactory therapeutic response or progression of aGvHD $(n=8)$, at the last study assessment only two were recorded as having no intestinal aGvHD involvement, suggesting that most had not responded to treatment at the time that they discontinued the study drug. 


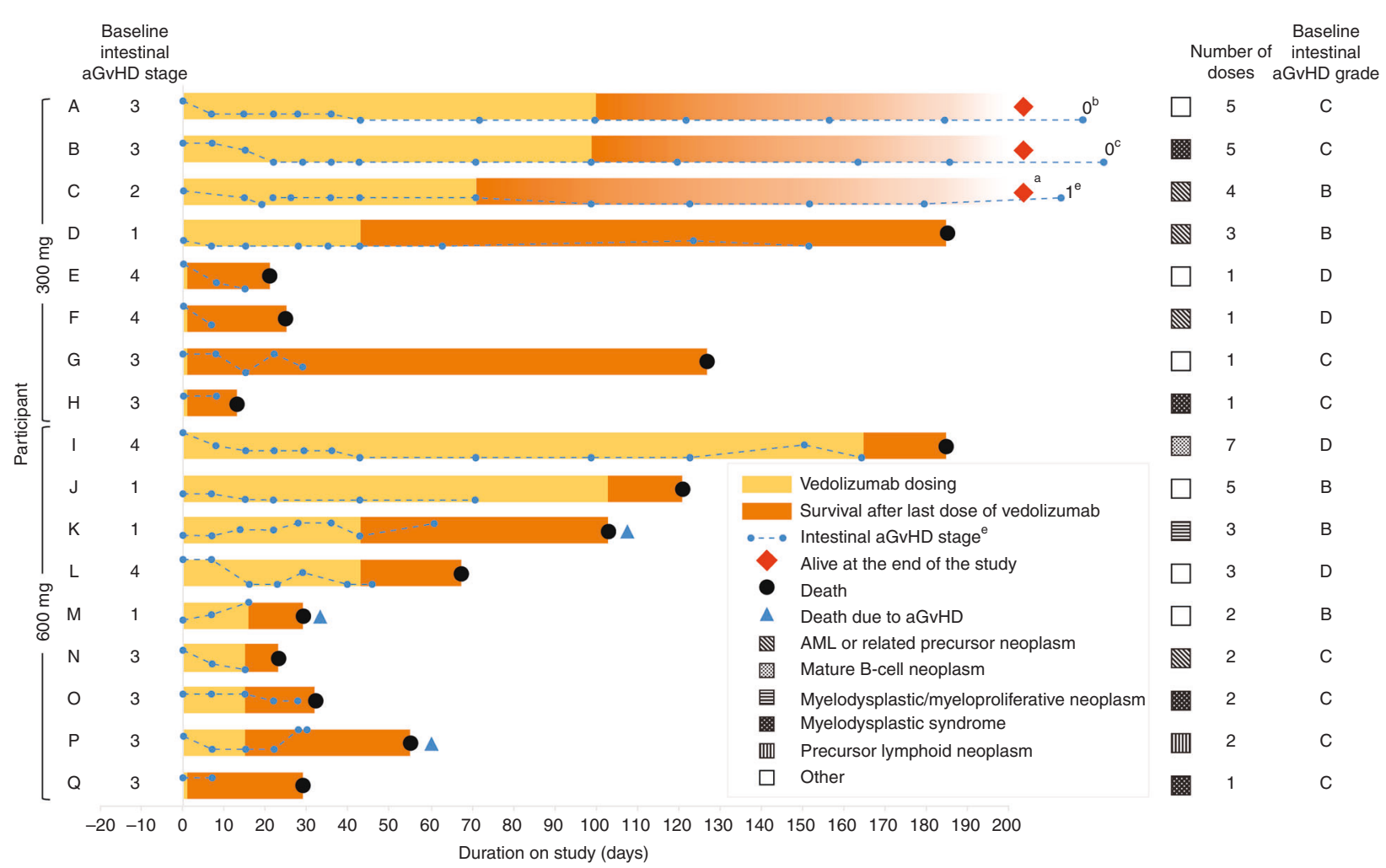

Fig. 2 Duration of vedolizumab treatment, survival following final vedolizumab dose, and participant characteristics. Data presented are from the efficacy analysis set. ${ }^{a}$ Intestinal aGvHD stages (based on aGvHD clinical stage criteria) are indicated by blue circles and dashed trace (uppermost circle aGvHD stage 4; lowermost circle aGvHD stage 0 ) positioned on the day that the aGvHD assessment was performed. Stage 0 no intestinal tract involvement, Stage $1>500 \mathrm{~mL}$ diarrhea/day,

\section{Overall response}

At day 28, a total of six participants (35.3\%) had an OR in all organs involved, and six of the eight participants with an OR in intestinal aGvHD at day 28 experienced a durable response (response maintained through day 43). An OR in intestinal aGvHD was recorded in 11 participants (64.7\%) and eight participants (47.1\%) at day 15 and day 28, respectively (Fig. 3). A complete response in all organs involved was reported in two participants at day 15 , in one at day 28 , and in four at day 43 .

\section{Overall survival}

At 6 months, five of the 17 participants (29.4\%) were alive: four $(50 \%$; 4 of 8$)$ in the vedolizumab $300 \mathrm{mg}$ and one $(11.1 \%$; 1 of 9$)$ in the vedolizumab $600 \mathrm{mg}$ group, with a median overall survival of 161 days (range, 16-310 days) in the $300 \mathrm{mg}$ group and 58 days (range, 24-189 days) in the $600 \mathrm{mg}$ group (Fig. 4). At the time of study termination, three participants were alive, all
Stage 2>1000 mL diarrhea/day, Stage 3>1500 mL diarrhea/day, Stage 4 severe abdominal pain with or without ileus. bday 255. 'day 276. ${ }^{\mathrm{d}}$ Participant $\mathrm{C}$ was alive at the end of the study and on day 239 experienced recurrence of intestinal aGvHD at Stage 1. ${ }^{\mathrm{e}}$ day 239. $a G v H D$ acute graft-versus-host disease, $A M L$ acute myeloid leukemia, $G v H D$ graft-versus-host disease.

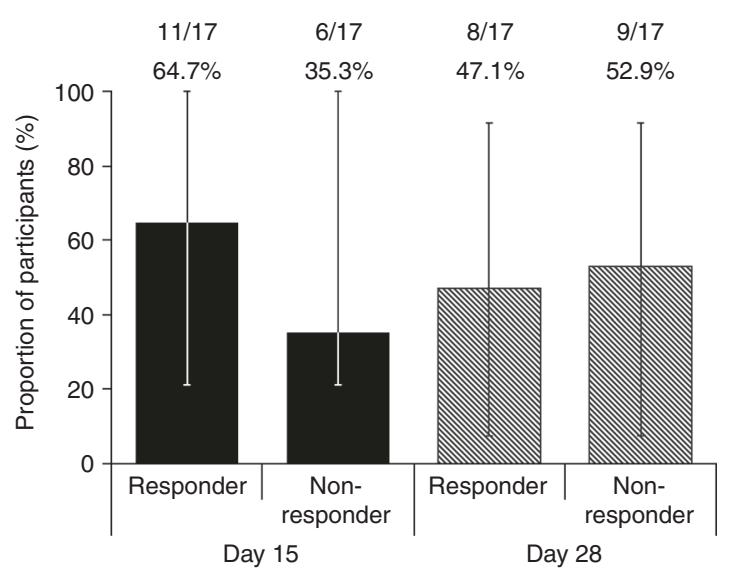

Fig. 3 Proportion of participants with intestinal overall response at day 15 and day 18. Error bars represent $95 \%$ confidence interval of percentage of responders. Participants with missing data for endpoint determination were categorized as non-responders.

from the $300 \mathrm{mg}$ group. Owing to the early termination of the study, overall survival at 12 months was not determined. 


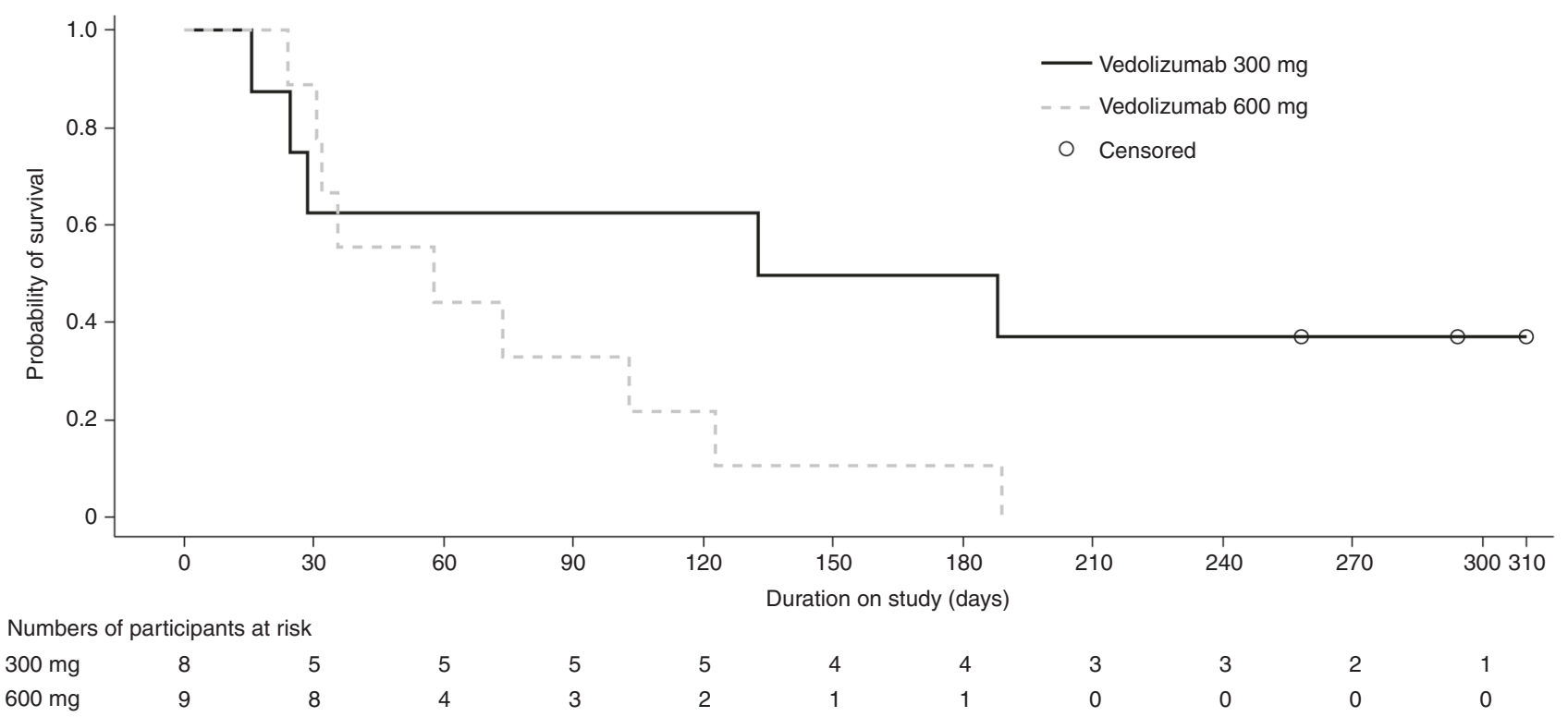

Fig. 4 Kaplan-Meier estimates of overall survival. Data presented are from the efficacy analysis set. Overall survival was defined as the time from the date of enrollment to the date of death. Participants

without documentation of death at the time of analysis were censored at the date last known to be alive.

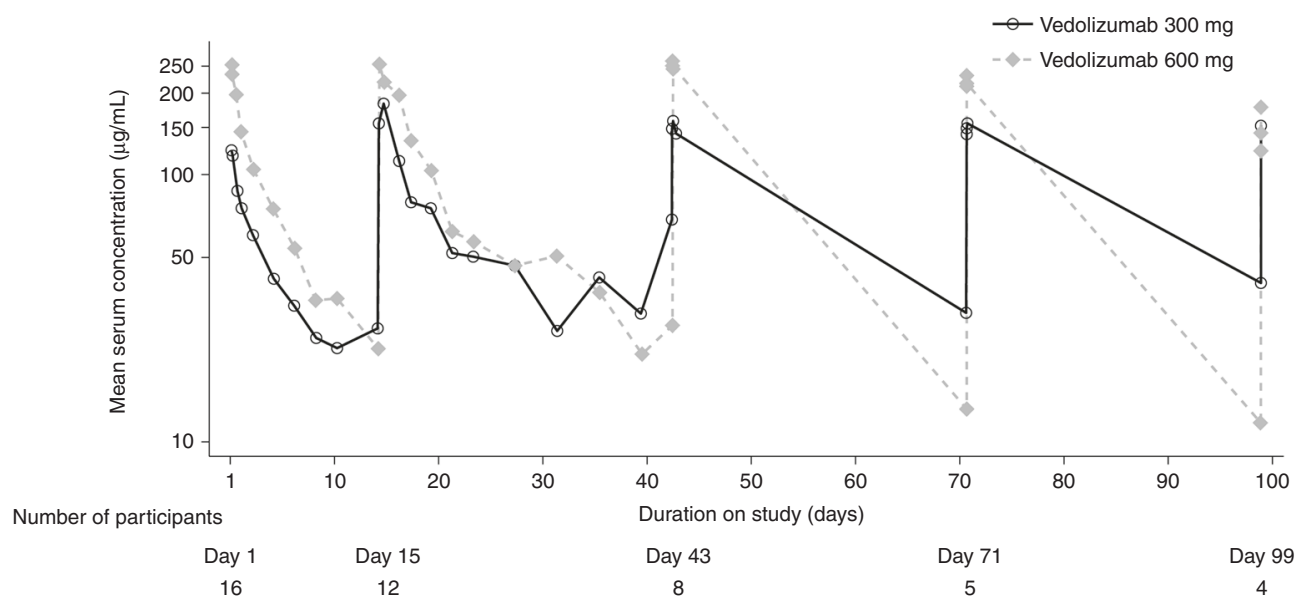

Fig. 5 Mean serum vedolizumab concentration-time profiles following multiple IV infusions of vedolizumab 300 or $600 \mathrm{mg}$ delivered over $30 \mathrm{~min}$ (semi-log scale). Data presented are from the pharmacokinetics analysis set $(n=16$, eight participants from 300 and

\section{Pharmacokinetics}

In general, exposure to vedolizumab was higher over time in participants receiving vedolizumab $600 \mathrm{mg}$ compared with those receiving vedolizumab $300 \mathrm{mg}$ and was comparable with that observed in previous multiple-dose PK studies in participants with ulcerative colitis and Crohn's disease $[31,32]$. Serum vedolizumab $C_{\max }$ was similar on all dosing days, whereas serum vedolizumab $C_{\text {trough }}$ was variable, with no clear trends over time or between groups (Fig. 5). $C_{\text {trough }}$ levels were lower in the $600 \mathrm{mg}$ group versus the $300 \mathrm{mg}$ group, but average $C_{\text {trough }}$ concentrations
$600 \mathrm{mg}$ dosing groups; participant $\mathrm{N}$ was excluded from the PK analysis set). The lower limit of quantification is $0.2 \mu \mathrm{g} / \mathrm{mL}$. The concentrations below the limit of quantification are included as 0 . Anomalous concentrations are excluded.

still met the target concentration level of $10 \mu \mathrm{g} / \mathrm{mL}$ for participants with inflammatory bowel diseases $[32,33]$.

\section{Safety, tolerability, and treatment-emergent adverse events}

An overview of TEAEs is presented in Supplementary Table 6. All participants in the study experienced at least one TEAE. A total of 50 serious adverse events were experienced by 15 participants $(88.2 \%)$, most of which $(n=46[92.0 \%])$ were considered unrelated to the study drug. Overall, five participants $(29.4 \%)$ experienced a 
TEAE, and two participants $(11.8 \%)$ experienced a serious adverse event considered to be related to the study drug.

The most frequently reported adverse events were infections occurring in 15 participants $(88.2 \%)$, including three participants $(17.6 \%)$ with sepsis and four participants (23.5\%) with cytomegalovirus (CMV) related infections, of which two (11.8\%) were CMV colitis. The events of CMV colitis were both serious and considered related to the study drug for one participant (vedolizumab $600 \mathrm{mg}$ ). Commonly occurring infection-related TEAEs are presented in Supplementary Table 7 .

No participants had detectable anti-vedolizumab antibodies at any scheduled time point.

\section{Deaths}

During the TEAE reporting period, 13 participants $(76.5 \%)$ died (Fig. 1). An additional participant died after the reporting period for TEAEs (18 weeks after the last dose of study drug). No deaths were considered by the investigator to be related to the study drug. The most common causes of death were attributed to complications associated progression of aGvHD $(n=5)$ or sepsis $(n=3)$. Other causes of death in the remaining participants $(n=5)$ who died during the TEAE period were due to the development of peritonitis (percutaneous endoscopic gastrostomy probe placement related), thrombotic microangiopathy, mediastinitis, or acute kidney injury; one cause of death was not reported. The death of the participant after the TEAE period was due to a fatal relapse of acute myeloid leukemia. Additional details at the time of death for each participant are provided in Table 2.

\section{Discussion}

The primary objective of this phase 2a study was to characterize the efficacy, tolerability, and safety of vedolizumab to identify a recommended dose for further evaluation of the treatment of SR intestinal aGvHD. While early clinical benefit $(64.7 \%$ OR rate at day 15) was observed in approximately two-thirds of participants, less than half obtained an OR in intestinal aGvHD by day 28, with a significant number $(n=8)$ of participants dying from complications of aGvHD. An early review (prior to day 28) of patient-level data for the 17 enrolled participants concluded that the expected efficacy outcome for the study would not be achieved and the decision was made to terminate the study prior to completion of planned enrollment.

There are a number of potential reasons for the lack of a satisfactory OR in this study. Most notably, all participants had SR aGvHD with lower intestinal involvement, with a strict definition of SR, and approximately $70 \%$ had severe
GI (Stage 3: 47.1\%; Stage 4: 23.5\%). Moreover, more than $70 \%$ of participants had SR Grade C or D aGvHD, and $\sim 30 \%$ had multiorgan involvement at baseline. Among patients with aGvHD intestinal tract involvement, GI Stage 3-4 and SR aGvHD in particular are associated with substantial morbidity and mortality, with a significantly increased risk of infections, and a limited response to salvage therapies [5, 34-38]. The significant complications inherent in patients with such disease, including early death and premature discontinuation of study drug, can possibly impair the ability to evaluate efficacy compared with other SR aGvHD studies, which have included patients without intestinal involvement.

Given the data from this study, it is also certainly possible that treatment with vedolizumab was unable to exert an effect rapid enough and/or of significant magnitude to allow the injured GI epithelium to recover sufficiently and prevent disease-related complications. It has been proposed that if enough tissue injury has occurred, T-cell migration to the GI endothelium is of limited importance, and $\alpha 4 \beta 7$ integrin is no longer needed for the propagation of aGvHD [39]. From this perspective, vedolizumab might not be expected to exert a significant therapeutic effect in advanced disease. This hypothesis is supported by a recent study by $\mathrm{Fu}$ et al., which highlighted the key role played by the $\alpha 4 \beta 7$-MAdCAM-1 interaction in the early recruitment of donor $\mathrm{T}$ cells to the intestinal stem cell compartment [40]. Therefore, vedolizumab may have greater therapeutic benefit if administered as initial or preventative therapy.

A number of case series on the use of vedolizumab in SR intestinal aGvHD have reported variable outcomes [9, 26, 39, 41, 42]. Fløisand et al. initially reported a $100 \%$ OR rate in six patients with SR intestinal aGvHD treated with vedolizumab $300 \mathrm{mg}$, with two deaths (due to sepsis/ acute respiratory distress syndrome and multiorgan failure) after a median follow-up time of 10 months [26]. Contrastingly, Bukauskas et al. reported five patients with SR aGvHD with intestinal involvement who were treated with vedolizumab and who died at a median of 32 days (range, 7-172 days) after starting therapy. All deaths were attributed to infectious complications with persistent aGvHD symptoms. Partial responses were observed in only two patients [41]. Similarly, Coltoff et al. reported high mortality rates in a retrospective case series of nine patients with SR intestinal aGvHD treated with vedolizumab $300 \mathrm{mg}$, with only one patient surviving beyond 60 days, with most patients dying of infection [42].

There have been two recent large retrospective multicenter case series published. Danylesko et al. described 29 patients with SR intestinal aGvHD treated with vedolizumab IV $300 \mathrm{mg}$ and demonstrated OR rates of $79 \%$ (28\% complete response, $52 \%$ partial response) after 7-10 days. Twelve patients $(41 \%)$ were able to discontinue 


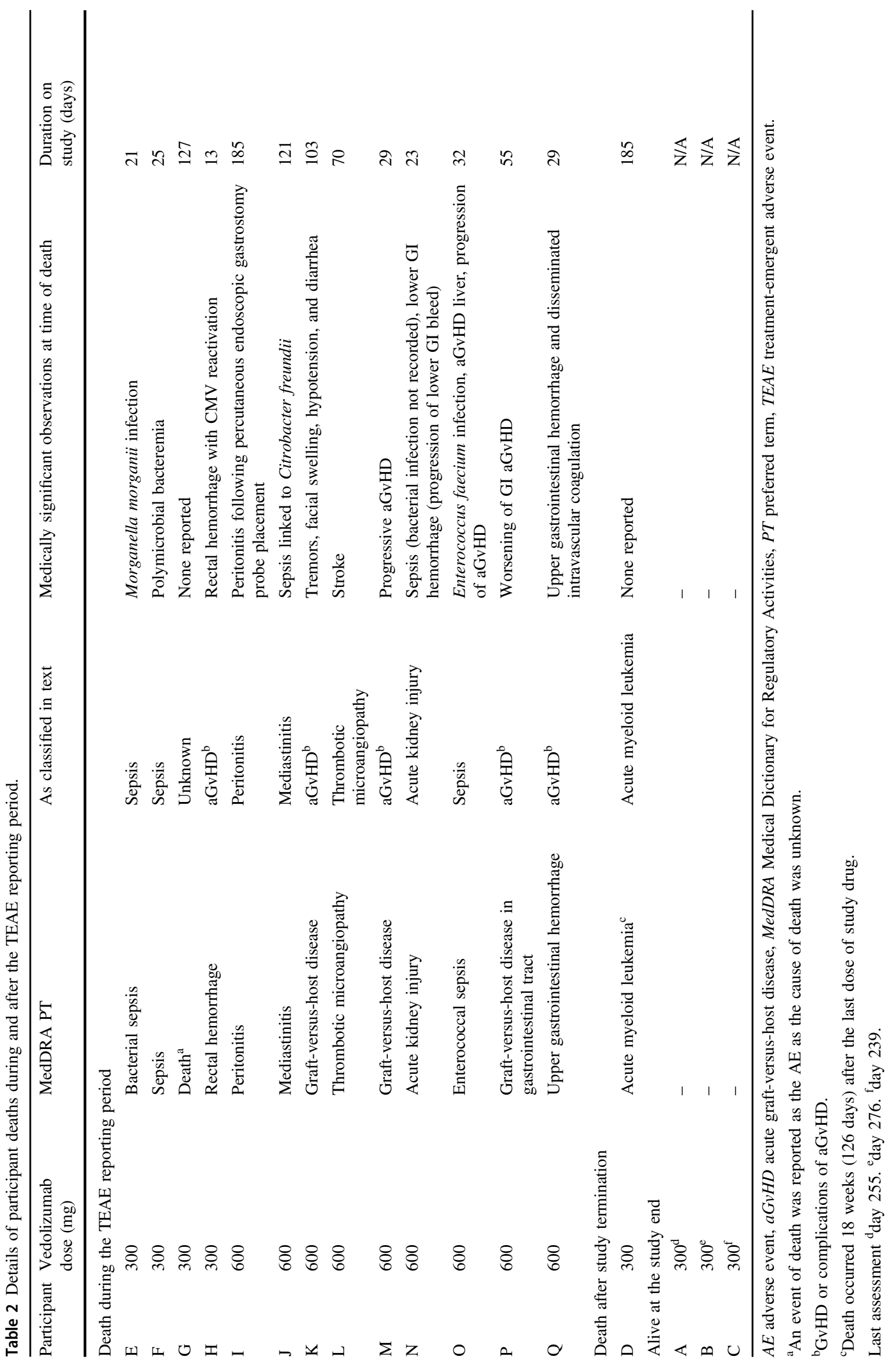


immunosuppressive therapy. Overall survival rates at 6 and 12 months were $41.4 \%$ and $27.5 \%$, respectively, with the majority of successful outcomes observed in patients who were treated early in their disease course [39]. Fløisand et al. described a series of 29 patients with SR aGvHD treated with vedolizumab, reporting an OR rate of $64 \%$ (6-10 weeks after initiation of drug) and a 6-month overall survival rate of $54 \%$ [9].

Taken together, the early OR results observed in this study, along with the anecdotal evidence from retrospective case studies, justify pursuing the evaluation of vedolizumab for lower intestinal SR GvHD. However, considering the mechanism of action and kinetics of GI epithelial regeneration in the context of aGvHD, vedolizumab may be more effective if administered earlier in the disease course, such as for prevention, pre-emptive use, or initial therapy.

With regard to safety, observed adverse events were comparable with prior reports in a patient population with SR GvHD [43]. The mortality rate observed in this trial was comparable to historical series of patients with SR aGvHD with intestinal involvement [44, 45]. In the current study, no safety signals were identified that were associated with the use of either dose of vedolizumab, and the reported adverse events were not related to immunogenicity because all participants were negative for anti-vedolizumab antibodies.

Overall, this study was unable to demonstrate a positive proof-of-concept in efficacy in participants with SR GvHD treated with vedolizumab. While early and durable responses to intestinal aGvHD following treatment with vedolizumab were observed in some participants, vedolizumab was unable to generate a satisfactory response in this study. Evaluating the true efficacy of vedolizumab in patients with advanced severe intestinal aGvHD and associated complications is clearly challenging and perhaps not realistically possible. It may be that vedolizumab may be more suited to use for intestinal GvHD prevention, and this is currently being evaluated in a phase 3 clinical trial (NCT03657160).

The observations made in this study are nevertheless important, as they provide vital information on the severity of SR intestinal aGvHD and management of complications that need to be considered when designing and conducting future studies in such patients if positive efficacy outcomes are to be demonstrated.

\section{Data availability}

Data pertaining to this study and the associated study protocol may be found at ClinicalTrials.gov (identifier: NCT02993783). Reuse of these data is not permitted. Additional data that support the findings of this study are available from Takeda Pharmaceuticals International upon reasonable request.
Acknowledgements This study was sponsored by Takeda Pharmaceutical Company Ltd. Medical writing assistance was provided by Steve Banner of Oxford PharmaGenesis, Oxford, UK and was supported by Takeda Pharmaceutical Company, Ltd.

\section{Compliance with ethical standards}

Conflict of interest YF: consultancy fees from Takeda, Celgene, Novartis, Pfizer, Otsuka and Astellas (advisory boards and speaker). MAS: advisory board and received honorary or consultant fees from (year of last engagement): Amgen (2017), Astellas (Feb 2019), Dova pharmaceuticals (May 2019), FlatIron Inc (June 2019), GSK (October 2019), Gilead Sciences (2017), Incyte (May 2018), Novo Nordisk (2018), Partners Therapeutics (Nov 2018), Pfizer (July 2018), Sanofi Genzyme (2017). Speaker bureau (all terminated August 2019): Abbvie, Merck, Takeda. Research funding: Incyte, Seattle Genetics, Janssen, Genzyme Sanofi, Celgene. PC, DS, SD, ASR: nothing to disclose. MM has received fees for consultancy and speaker bureau participation, honoraria and research funding from Sanofi, Janssen, Amgen, Takeda, Celgene, GSK, Jazz, Adaptive, Stemline, BMS, and Novartis. IYA received honorarium from Takeda, Celgene, Novartis, Janssen, Gilead/Kite. CC was an employee of Millennium Pharmaceuticals Inc. at the time the study was performed. AP was an employee of Millennium Pharmaceuticals Inc. at the time the study was performed. He is currently the Associate Director of Biostatistics at Sage Therapeutics. SQ and JJ are employees of Millennium Pharmaceuticals Inc. MA was a Takeda employee at the time the study was performed; She is currently a Senior Medical Director at AbbVie. YBC: consulting fees for Takeda, Magenta, Abbvie, Daiichi, Equilium, Incyte, Pharmacyclics, Celularity.

Ethical approval This study was conducted in accordance with Good Clinical Practice regulations and guidelines, the Declaration of Helsinki, and the International Council for Harmonisation of Technical Requirements for Pharmaceuticals for Human Use, in addition to all applicable local or regional regulatory requirements [29, 30]. Relevant documentation was supplied to the IRB or IEC for review and approval of the protocol.

Publisher's note Springer Nature remains neutral with regard to jurisdictional claims in published maps and institutional affiliations.

Open Access This article is licensed under a Creative Commons Attribution 4.0 International License, which permits use, sharing, adaptation, distribution and reproduction in any medium or format, as long as you give appropriate credit to the original author(s) and the source, provide a link to the Creative Commons license, and indicate if changes were made. The images or other third party material in this article are included in the article's Creative Commons license, unless indicated otherwise in a credit line to the material. If material is not included in the article's Creative Commons license and your intended use is not permitted by statutory regulation or exceeds the permitted use, you will need to obtain permission directly from the copyright holder. To view a copy of this license, visit http://creativecommons. org/licenses/by/4.0/.

\section{References}

1. Ferrara JL, Levine JE, Reddy P, Holler E. Graft-versus-host disease. Lancet. 2009;373:1550-61.

2. Gratwohl A, Baldomero H, Passweg J, Frassoni F, Niederwieser D, Schmitz N, et al. Hematopoietic stem cell transplantation for 
hematological malignancies in Europe. Leukemia. 2003;17: 941-59.

3. Martin PJ, McDonald GB, Sanders JE, Anasetti C, Appelbaum $\mathrm{FR}$, Deeg HJ, et al. Increasingly frequent diagnosis of acute gastrointestinal graft-versus-host disease after allogeneic hematopoietic cell transplantation. Biol Blood Marrow Transplant. 2004;10:320-7.

4. Jacobsohn DA, Vogelsang GB. Acute graft versus host disease. Orphanet J Rare Dis. 2007;2:35.

5. Deeg HJ. How I treat refractory acute GVHD. Blood. 2007;109:4119-26.

6. Cesen Mazic M, Girandon L, Knezevic M, Avcin SL, Jazbec J. Treatment of severe steroid-refractory acute-graft-vs.-host disease with mesenchymal stem cells-single center experience. Front Bioeng Biotechnol. 2018;6:93.

7. Garnett C, Apperley JF, Pavlu J. Treatment and management of graft-versus-host disease: improving response and survival. Ther Adv Hematol. 2013;4:366-78.

8. Levine JE, Braun TM, Harris AC, Holler E, Taylor A, Miller H, et al. A prognostic score for acute graft-versus-host disease based on biomarkers: a multicentre study. Lancet Haematol. 2015;2:e21-29.

9. Fløisand Y, Lazarevic VL, Maertens J, Mattsson J, Shah NN, Zachee $\mathrm{P}$, et al. Safety and effectiveness of vedolizumab in patients with steroid-refractory gastrointestinal acute graft-versushost disease: a retrospective record review. Biol Blood Marrow Transplant. 2019;25:720-7.

10. Zeiser R, Burchert A, Lengerke C, Verbeek M, Maas-Bauer K, Metzelder SK, et al. Ruxolitinib in corticosteroid-refractory graftversus-host disease after allogeneic stem cell transplantation: a multicenter survey. Leukemia. 2015;29:2062-8.

11. Munneke JM, Spruit MJ, Cornelissen AS, van Hoeven V, Voermans C, Hazenberg MD. The potential of mesenchymal stromal cells as treatment for severe steroid-refractory acute graft-versushost disease: a critical review of the literature. Transplantation. 2016;100:2309-14.

12. Incyte. Prescribing Information JAKAFI ${ }^{\circledR}$ (ruxolitinib) [Wilmington, DE]. Food and Drug Administration; 2011.

13. Hill GR, Ferrara JL. The primacy of the gastrointestinal tract as a target organ of acute graft-versus-host disease: rationale for the use of cytokine shields in allogeneic bone marrow transplantation. Blood. 2000;95:2754-9.

14. Takeda Pharmaceuticals America, Inc. Prescribing Information ENTYVIO $^{\circledast}$ (vedolizumab) [Deerfield, IL]. Food and Drug Administration; 2014.

15. Wyant T, Fedyk E, Abhyankar B. An overview of the mechanism of action of the monoclonal antibody vedolizumab. J Crohns Colitis. 2016;10:1437-44.

16. Takeda Pharma A/S. Summary of Product Characteristics ENTYVIO $^{\circledR}$ (vedolizumab) [Vallensbaek Strand, Denmark]. European Medicines Agency; 2018.

17. Butcher EC, Williams M, Youngman K, Rott L, Briskin M. Lymphocyte trafficking and regional immunity. Adv Immunol. 1999;72:209-53.

18. Salmi M, Jalkanen S. Lymphocyte homing to the gut: attraction, adhesion, and commitment. Immunol Rev. 2005;206:100-13.

19. Erle DJ, Briskin MJ, Butcher EC, Garcia-Pardo A, Lazarovits AI, Tidswell M. Expression and function of the MAdCAM-1 receptor, integrin alpha 4 beta 7 , on human leukocytes. J Immunol. 1994;153:517-28.

20. Briskin M, Winsor-Hines D, Shyjan A, Cochran N, Bloom S, Wilson J, et al. Human mucosal addressin cell adhesion molecule1 is preferentially expressed in intestinal tract and associated lymphoid tissue. Am J Pathol. 1997;151:97-110.

21. Chen YB, Kim HT, McDonough S, Odze RD, Yao X, Lazo-Kallanian S, et al. Up-regulation of alpha4beta7 integrin on peripheral $\mathrm{T}$ cell subsets correlates with the development of acute intestinal graft-versus-host disease following allogeneic stem cell transplantation. Biol Blood Marrow Transplant. 2009; 15:1066-76.

22. Ueha S, Murai M, Yoneyama H, Kitabatake M, Imai T, Shimaoka $\mathrm{T}$, et al. Intervention of MAdCAM-1 or fractalkine alleviates graft-versus-host reaction associated intestinal injury while preserving graft-versus-tumor effects. J Leukoc Biol. 2007;81: 176-85.

23. Waldman E, Lu SX, Hubbard VM, Kochman AA, Eng JM, Terwey $\mathrm{TH}$, et al. Absence of beta7 integrin results in less graftversus-host disease because of decreased homing of alloreactive T cells to intestine. Blood. 2006;107:1703-11.

24. Ward MG, Sparrow MP, Roblin X. Therapeutic drug monitoring of vedolizumab in inflammatory bowel disease: current data and future directions. Ther Adv Gastroenterol. 2018;11: 1756284818772786.

25. Rezvani AR, Storer BE, Storb RF, Mielcarek M, Maloney DG, Sandmaier BM, et al. Decreased serum albumin as a biomarker for severe acute graft-versus-host disease after reduced-intensity allogeneic hematopoietic cell transplantation. Biol Blood Marrow Transplant. 2011;17:1594-601.

26. Fløisand Y, Lundin KEA, Lazarevic V, Kristiansen JD, Osnes LTN, Tjonnfjord GE, et al. Targeting integrin alpha4beta7 in steroid-refractory intestinal graft-versus-host disease. Biol Blood Marrow Transplant. 2017;23:172-5.

27. Przepiorka D, Weisdorf D, Martin P, Klingemann HG, Beatty P, Hows J, et al. 1994 consensus conference on acute GvHD grading. Bone Marrow Transplant. 1995;15:825-8.

28. Rowlings PA, Przepiorka D, Klein JP, Gale RP, Passweg JR, Henslee-Downey PJ, et al. IBMTR Severity Index for grading acute graft-versus-host disease: retrospective comparison with Glucksberg grade. Br J Haematol. 1997;97:855-64.

29. Singh J. International conference on harmonization of technical requirements for registration of pharmaceuticals for human use. $\mathrm{J}$ Pharmacol Pharmacother. 2015;6:185-7.

30. World Medical Association. World Medical Association Declaration of Helsinki: ethical principles for medical research involving human subjects. JAMA. 2013;310:2191-4.

31. Parikh A, Leach T, Wyant T, Scholz C, Sankoh S, Mould DR, et al. Vedolizumab for the treatment of active ulcerative colitis: a randomized controlled phase 2 dose-ranging study. Inflamm Bowel Dis. 2012;18:1470-9.

32. Rosario M, Dirks NL, Milch C, Parikh A, Bargfrede M, Wyant T, et al. A review of the clinical pharmacokinetics, pharmacodynamics, and immunogenicity of vedolizumab. Clin Pharmacokinet. 2017;56:1287-301.

33. Rosario M, Wyant T, Leach T, Sankoh S, Scholz C, Parikh A, et al. Vedolizumab pharmacokinetics, pharmacodynamics, safety, and tolerability following administration of a single, ascending, intravenous dose to healthy volunteers. Clin Drug Investig. 2016;36:913-23.

34. Leslie K, Stonell CA. Anaesthesia and sedation for gastrointestinal endoscopy. Curr Opin Anaesthesiol. 2005;18:431-6.

35. Murray JA, Rubio-Tapia A, Van Dyke CT, Brogan DL, Knipschield MA, Lahr B, et al. Mucosal atrophy in celiac disease: extent of involvement, correlation with clinical presentation, and response to treatment. Clin Gastroenterol Hepatol. 2008;6: 186-93. quiz 125.

36. Holtan SG, Pasquini M, Weisdorf DJ. Acute graft-versus-host disease: a bench-to-bedside update. Blood. 2014;124:363-73.

37. MacMillan ML, Weisdorf DJ, Wagner JE, DeFor TE, Burns LJ, Ramsay NK, et al. Response of 443 patients to steroids as primary therapy for acute graft-versus-host disease: comparison of grading systems. Biol Blood Marrow Transplant. 2002;8:387-94.

38. MacMillan ML, DeFor TE, Weisdorf DJ. What predicts high risk acute graft-versus-host disease (GVHD) at onset?: identification of 
those at highest risk by a novel acute GVHD risk score. Br J Haematol. 2012;157:732-41.

39. Danylesko I, Bukauskas A, Paulson M, Peceliunas V, Gedde-Dahl DYT, Shimoni A, et al. Anti-alpha4beta7 integrin monoclonal antibody (vedolizumab) for the treatment of steroid-resistant severe intestinal acute graft-versus-host disease. Bone Marrow Transplant. 2018;54:987-93.

40. Fu YY, Egorova A, Sobieski C, Kuttiyara J, Calafiore M, Takashima $S$, et al. T cell recruitment to the intestinal stem cell compartment drives immune-mediated intestinal damage after allogeneic transplantation. Immunity. 2019;51:90-103. e103

41. Bukauskas A, Griskevicius L, Peceliunas V. Lessons learned from early experiences with vedolizumab for steroid-refractory acute graft- versus-host disease with gastrointestinal involvement. Biol Blood Marrow Transplant. 2017;23:1597.
42. Coltoff A, Lancman G, Kim S, Steinberg A. Vedolizumab for treatment of steroid-refractory lower gastrointestinal acute graftversus-host disease. Bone Marrow Transplant. 2018;53:900-4.

43. Xhaard A, Rocha V, Bueno B, de Latour RP, Lenglet J, Petropoulou A, et al. Steroid-refractory acute GVHD: lack of long-term improved survival using new generation anticytokine treatment. Biol Blood Marrow Transplant. 2012;18:406-13.

44. Castilla-Llorente C, Martin PJ, McDonald GB, Storer BE, Appelbaum FR, Deeg HJ, et al. Prognostic factors and outcomes of severe gastrointestinal GVHD after allogeneic hematopoietic cell transplantation. Bone Marrow Transplant. 2014;49:966-71.

45. Leonard MM, Sapone A, Catassi C, Fasano A. Celiac disease and nonceliac gluten sensitivity: a review. JAMA. 2017;318:647-56. 